\title{
Stress-Limiting Test Structures for Rapid Low-Cost Strength and Stiffness Assessment
}

\author{
Andrew Katz \\ University of South Florida \\ Craig P. Lusk \\ University of South Florida \\ Nathan B. Crane \\ Brigham Young University - Provo, nbcrane@byu.edu
}

Follow this and additional works at: https://scholarsarchive.byu.edu/facpub

Part of the Manufacturing Commons, and the Other Mechanical Engineering Commons

\section{Original Publication Citation}

A. Katz, J. Nussbaum, C. P. Lusk, and N. B. Crane, "Stress-Limiting Test Structures for Rapid Low-Cost Strength and Stiffness Assessment," Rapid Prototyping Journal, Vol 21 (2015) pp 144-151 (DOI: 10.1108/RPJ-12-2014-0170).

\section{BYU ScholarsArchive Citation}

Katz, Andrew; Lusk, Craig P.; and Crane, Nathan B., "Stress-Limiting Test Structures for Rapid Low-Cost Strength and Stiffness Assessment" (2015). Faculty Publications. 5344.

https://scholarsarchive.byu.edu/facpub/5344

This Peer-Reviewed Article is brought to you for free and open access by BYU ScholarsArchive. It has been accepted for inclusion in Faculty Publications by an authorized administrator of BYU ScholarsArchive. For more information, please contact ellen_amatangelo@byu.edu. 


\title{
STRESS-LIMITING TEST STRUCTURES FOR RAPID LOW-COST STRENGTH AND STIFFNESS ASSESSMENT
}

\author{
Andrew Katz, Justin Nussbaum, Craig P. Lusk and Nathan B. Crane \\ Department of Mechanical Engineering, University of South Florida, Tampa, Florida, USA
}

\begin{abstract}
Purpose: Evaluate the use of a simple printed geometry to estimate mechanical properties (elastic modulus, yield strength) with inexpensive test equipment.

Design Methodology/Approach: Test geometry is presented that enables controlled strains with manual deformation and repeatable measurement of vibrational frequencies. This is tested with multiple FDM machines to assess measurement accuracy and repeatability. Printing orientation and some printing parameters are varied to assess the measurement sensitivity.
\end{abstract}

Findings: The test methods show good correlation with manufacturer material specifications in the $\mathrm{X}-\mathrm{Y}$ plane and reported elastic strain limits. It is also sensitive to printing orientation and printing parameters.

Research Limitations/Implications: Further work is needed to assess the sensitivity of the method to particular defects and parameter errors expected in particular applications.

Originality/Value: This method supports process monitoring in production environments and inexpensive assessments of material properties for hobbyist and DIY users. While it is tested with FDM, it should be applicable to other AM processes.

KEYWORDS: Yield Strength, Elastic Modulus, FDM, process monitoring, additive manufacturing, 3D printing

\section{Introduction}

Additive manufacturing (AM) refers to processes that build up parts by adding material under computer control. This enables the fabrication of a wide variety of different geometries with drastically reduced costs of variety [1]. However, the process does not just form geometry, it forms a material locally. In many processes, the material properties are sensitive to the processing methods and may vary with parameters such as position, orientation and ambient conditions to name a few [2-6]. As AM processes move from making interesting geometries, to producing functional parts and systems, the control of these properties becomes increasingly important [7]. To address this challenge, there is an increasing focus on process monitoring and control, particularly online process monitoring $[8,9]$. 
There is also a need for effective monitoring of the finished products. The objective of this work is to describe a low cost means of evaluating the modulus (resistance to deflection) and strength (resistance to failure) of thin beams printed using Additive Manufacturing (AM) processes. To this end, we describe two tests, the first of which, the stiffness test, is non-destructive, and the second, the strength test, when conclusive, results in fracture or permanent deformation of the part. Both tests require limited or no specialized measurement equipment and can be performed using a simple printed structure illustrated in Figure 1.

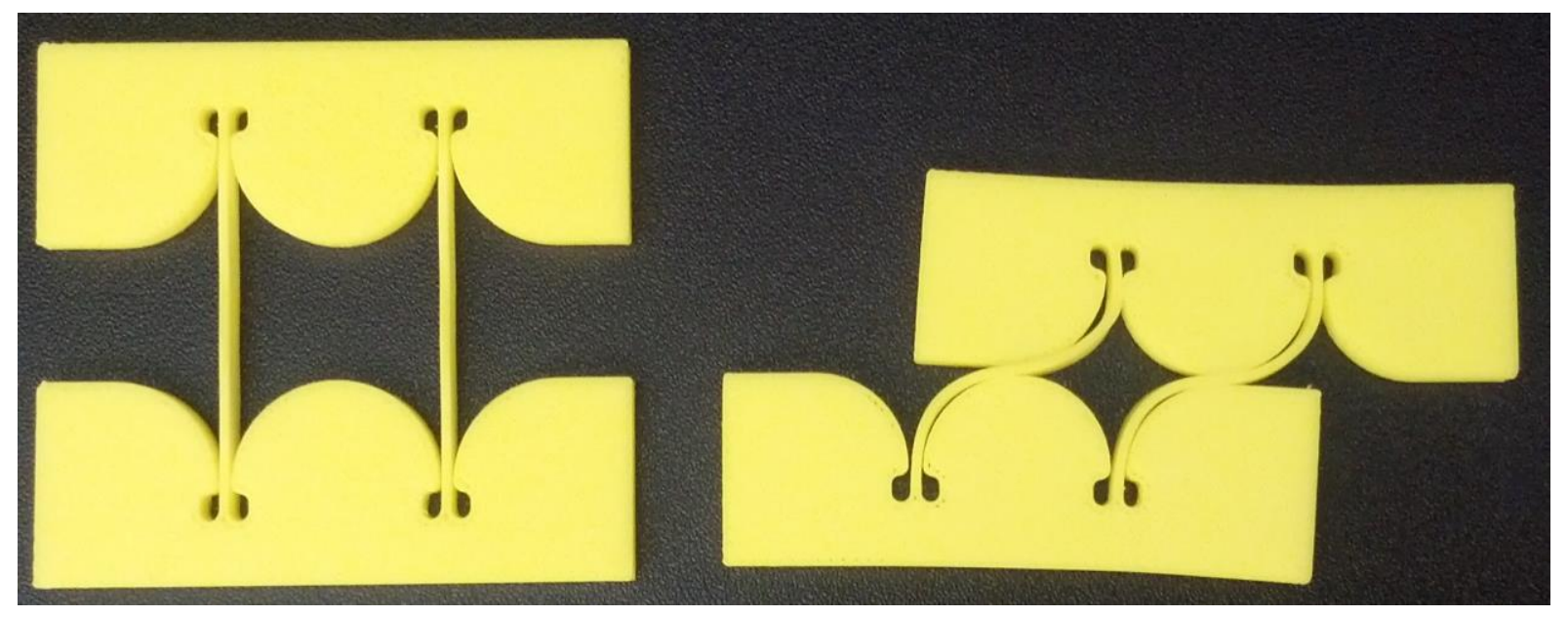

Figure 1 Photo of the basic test geometry in its "as-printed" (left) and deformed (right) positions.

These tests are intended to complement and perhaps substitute for the standard tensile and flexural bending tests which are performed using immobile and expensive tensile testing equipment. They may also have some utility in process control or material evaluation, in that they capture the key performance measures for structural integrity in simple, inexpensive tests. Thus, researchers seeking to improve the properties of an AM material could use these tests to ensure that they had not seriously compromised the structural utility of the material. The test method could be applied in production as an inexpensive method of verifying the stability of the production process. It may also provide a simple way for Do It Yourself (DIY) 3-D printing enthusiasts to assess the quality and stability of their processes using equipment they already have available.

The theory on which the measurement method is based is reviewed below. The subsequent sections describe how this design was implemented and tested using thermal extrusion processes. Stress test results are reported for a series of parts produced on a Stratasys Dimension 768 system. Stiffness measurements are reported for parts produced on two different thermal extrusion systems: RepStrap, and the Dimension 768. All tests were done using Acrylonitrile Butadiene Styrene (ABS) materials, each with its own feedstock.

\section{Theory}

We first describe the theoretical basis for the stiffness test. Our measurement method is based on measuring the natural frequency of a printed test structure. Vibrational frequencies of cantilever beams have previously been used to measure the modulus of elasticity of a variety of materials using both force and optical sensing methods [10-12]. This method has been covered in detail by ASTM standard E1876-01 [13]. This method requires only the test part, a striking mechanism such 
as a hammer and frequency sensing device, which could be a smart phone with an accelerometer or a microphone depending on the material and natural frequency of the part.

In order for a vibration to occur, there must be a disturbance of some of the mass in a system from equilibrium. Further, that system must be capable of producing a restoring force that drives the mass back to its equilibrium position. The interaction of the restoring force and the mass's inertia produce oscillatory motion about the equilibrium position of the mass. In its simplest form, the location of the mass is described by a position coordinate, $x(t)$, as a function of time. The differential equation describing the moving mass, $m$, of the system is given by:

$$
m \ddot{x}+F(x)=0
$$

When the displacement of the mass is small, and the mass does not rotate, the restoring force, $F(x)$, can often be written as a linear function of the position. Thus, when the equilibrium position is taken as the origin for the $x$-coordinate, the governing equation is:

$$
m \ddot{x}+k x=0
$$

where $k$ is a measure of the stiffness of the system. The solution to equation (2) is given by equations of the form:

$$
x(t)=A \cos (\sqrt{k / m} t+\varphi)
$$

where $\mathrm{A}$, and $\varphi$ are the amplitude and phase respectively, and their values depend on the details of the system's disturbance from equilibrium. On the other hand, the frequency of the oscillation of the system, $\sqrt{\mathrm{k} / \mathrm{m}}$, only depends on the system stiffness and moving mass. For our test, the moving mass is estimated as half of the total mass of the printed part, based on the symmetry between the fixed lower half and the moving upper half. Thus, by measuring the vibration frequency, $\omega$, of the part when it is tapped lightly, we can use the part's moving mass, $m$, to determine its stiffness:

$$
k=m \omega^{2}
$$

The stiffness value, $k$, is specific to a given geometry and material, and can be used to track the repeatability of an AM machine's building of a target geometry.

The governing equation (2) neglects energy losses from the system, and so equation (3) predicts that the vibrations will occur ad infinitum at the same magnitude. Of course this never happens, and the length of time that a part vibrates is indicative of the speed at which energy is lost from the system as heat or sound. The exclusion of such damping effects means that equation (4) tends to slightly over-predict the stiffness of a system. The amount of over-prediction depends on the internal damping in the material. For example, we were able to observe thirty periods of oscillation in an ABS part but only eight periods of oscillation in an acrylic part with the same geometry. Thus, we would assume that there is lower damping in the ABS, and the stiffness value obtained using equation (4) is closer to correct for the ABS part than for the acrylic one.

In the parts we were testing, the restoring force was provided by two identical thin flexures undergoing bending when the moving mass was plucked. Based on the dimensional considerations, we can surmise that the first bending mode with the lowest frequency is the 
fundamental vibration mode. Additionally we can conclude that based on the physics of the problem and the necessity for consistent units that the stiffness of the beam can be related to geometric and material parameters as follows:

$$
k=a E I / l
$$

where $E$ is the tensile modulus, $I$ is the second moment of area, and $l$ is the length of the beam. The proportional constant, $a$, is a dimensionless number that is specific to the shape of the part. Theoretical values for $a$ have been tabulated for various ideal loading conditions [14], but these are difficult to relate effectively to actual parts because the proportional constant is quite sensitive to the specifics of how the beams are attached to the moving and fixed portions of the system. However, the constant, $a$, can be measured experimentally or predicted using Finite Element Analysis (FEA).

It may be desirable to compare the stiffness results of two parts which are printed from the same Computer-Aided Drawing (CAD) file using different process parameters or printing orientations. In this geometry, the stiffness will be particularly sensitive to the width of the flexible segments. Variations in other dimensions and the internal fill structure will be reflected in the overall weight of the part. The reference part could be a part of known properties or it could be the as-designed component using frequencies predicted analytically and calculated part mass. Taking the reference part as having mass, $m_{0}$, and stiffness, $k_{0}$, and the new part having mass, $m_{l}$, and stiffness $k_{l}$, we can define:

$$
\alpha=m_{1} / m_{0}
$$

and

$$
\beta=k_{1} / k_{0}
$$

From equation (4), the ratio of vibration frequencies of the two parts is:

$$
\frac{\omega_{1}}{\omega_{0}}=\sqrt{\frac{\beta}{\alpha}}
$$

Using equation (5), taking the second moment of area for a rectangular beam, $I=w^{3} t / 12$, and canceling the part thickness $t$, we can write equation (7) as:

$$
\beta=\left(E_{1} w_{1}^{3}\right) /\left(E_{0} w_{0}^{3}\right)
$$

Then solving equations (6-9) for the ratio of moduli, we can see if process changes have affected the bulk stiffness of the material, by calculating:

$$
E_{1} / E_{0}=\left(m_{1} \omega_{1}^{2} w_{0}^{3}\right) /\left(m_{0} \omega_{0}^{2} w_{1}^{3}\right)
$$

The second test measures the strength of the material used in a bending test. This test does not measure the strength directly but is based on the relationships between bending and internal moment, and internal moment and stress. The Bernoulli-Euler hypothesis asserts that the relationship between bending and the internal moment is: 


$$
M(s)=E I / R(s)
$$

or in words, the moment at a point, $s \varepsilon(0, l)$, along a beam is equal to the beam stiffness, $E I$, divided by its radius of curvature (change in radius of curvature if initially curved). The stress due to bending is:

$$
\sigma=M y / I
$$

where $y$ is the distance from the neutral axis of the beam, which is a maximum at a distance $c=$ $w / 2$, i.e. half the width of the beam.

Putting equations (11) and (12) together, and rearranging one finds that:

$$
\sigma / E=c / R
$$

In other words, the ratio of stress to modulus equals the ratio of half the width of the beam to the radius of curvature of the beam. This means that if a beam is bent across a series of circular cams (different $R$ values), the stress in the beam will increase as the radii of the circular cams decrease. There is a certain radius cam for which the beam will experience its fracture stress or a $0.2 \%$ offset yield strength (i.e. the stress which causes $0.2 \%$ permanent strain in the beam). These failure stresses can therefore be associated with specific geometries or cam radii, which can be fabricated as the test geometry. If the beam can be bent ninety degrees around the cam without experiencing failure, the beam is stronger than expected. Thus, by building and testing parts with decreasing test radii, increasingly severe stress tests may be imposed on the material. As equation (8) associates a specific stress with a given cam radii (assuming that the modulus is known), the tests allow bounds on the fracture stress to be found.

The situation is more complicated for materials with beams that do not fracture when bent across cams of the smallest feasible size. For example, we found using ABS beams of one millimeter width that we saw increasing amounts of plastic deformation but that fracturing a thin beam

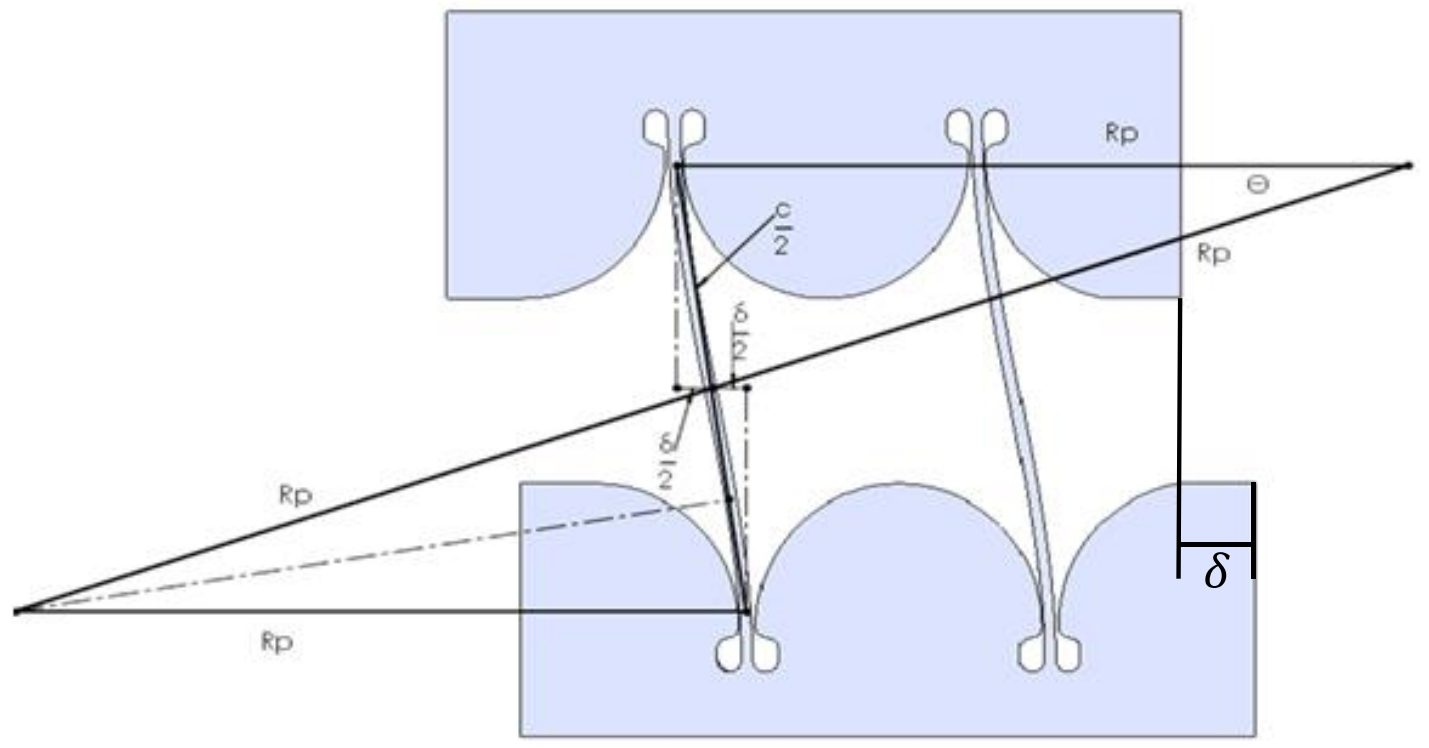

Figure 2 Geometry of the deformed test structure. 
required a $180^{\circ}$ fold of minimal radius. When beams undergo plastic deformation but do not fracture, we observe that the stress in the beam is constant in the section that is in contact with the cam. We can therefore assume that the amount of residual plastic strain, $\varepsilon_{p}$, in the beam is constant along each half of the beam (one half of the beam is bent across the bottom cam and the other half is bent in the opposite direction across the top cam). Thus, each half of the beam is deformed into a circular arc, with radius $R_{p}$, subtended $\operatorname{arc} \theta$, and arc-length $L / 2$.

Using the geometry of circular arcs, we find:

$$
R_{p} \theta=L / 2
$$

and

$$
R_{p}(1-\cos (\theta))=\delta / 2
$$

where $\delta$ is the horizontal translation of the top of the part relative to the bottom caused by the plastic deformation of the thin beams. This can be related to the residual plastic strain in the fibers of the beam by particularizing equation (13) as follows:

$$
\varepsilon_{p}=\sigma / E=c / R_{p}
$$

By combining equations (14-16), we can express $\delta$ in terms of the residual strain, and the beam width and length as:

$$
\delta=\frac{w}{\varepsilon_{p}}\left(1-\cos \left(\frac{L \varepsilon_{p}}{w_{b}}\right)\right)
$$

Equation (17) can be used to solve for the residual strain, when the deformed horizontal translation is known. The displacement associated with a $0.2 \%$ strain is:

$$
\delta=500 w\left(1-\cos \left(\frac{L}{500 w}\right)\right)
$$

On the other hand, we can compare the residual strain to the applied strain, which comes from bending the beams over the cams. The radius of curvature of the beams is:

$$
R_{d}=R+c
$$

Where $R_{d}$ is the radius of curvature of the beam, $R$ is the radius of the cam, and $c$ is half the width of the beam. The applied strain in the beam is:

$$
\varepsilon_{a}=\sigma / E=c / R_{d}
$$

The strain recovered when the beam is unloaded is

$$
\varepsilon_{r}=\varepsilon_{a}-\varepsilon_{p}
$$




\section{Specimen Design}

The test part is designed to leverage these pure bending effects possible with a cam to create a defined loading condition without requiring expensive testing equipment. Figure 3 defines the key parameters in the specimen design. The top and bottom rectangular sections provide both a solid base for attachment and a mass that drives the oscillation. Equation (13) was used to calculate what the radius should be. The beam width, $2 c$, was selected based on the size of the feature we wished to test. These tests were done with a width of one millimeter. It should be noted that failure modes in AM parts can vary with size, but a smaller value of beam width $(2 c)$ will result in smaller test structures. This indicates what the cam radius should be to cause the beam to yield when bent across it. Using these basic relationships, a radius and beam width $(c)$ can be selected that will evaluate the minimum strength of the specimen. The strength can be bracketed by testing a range of different specimen sizes.

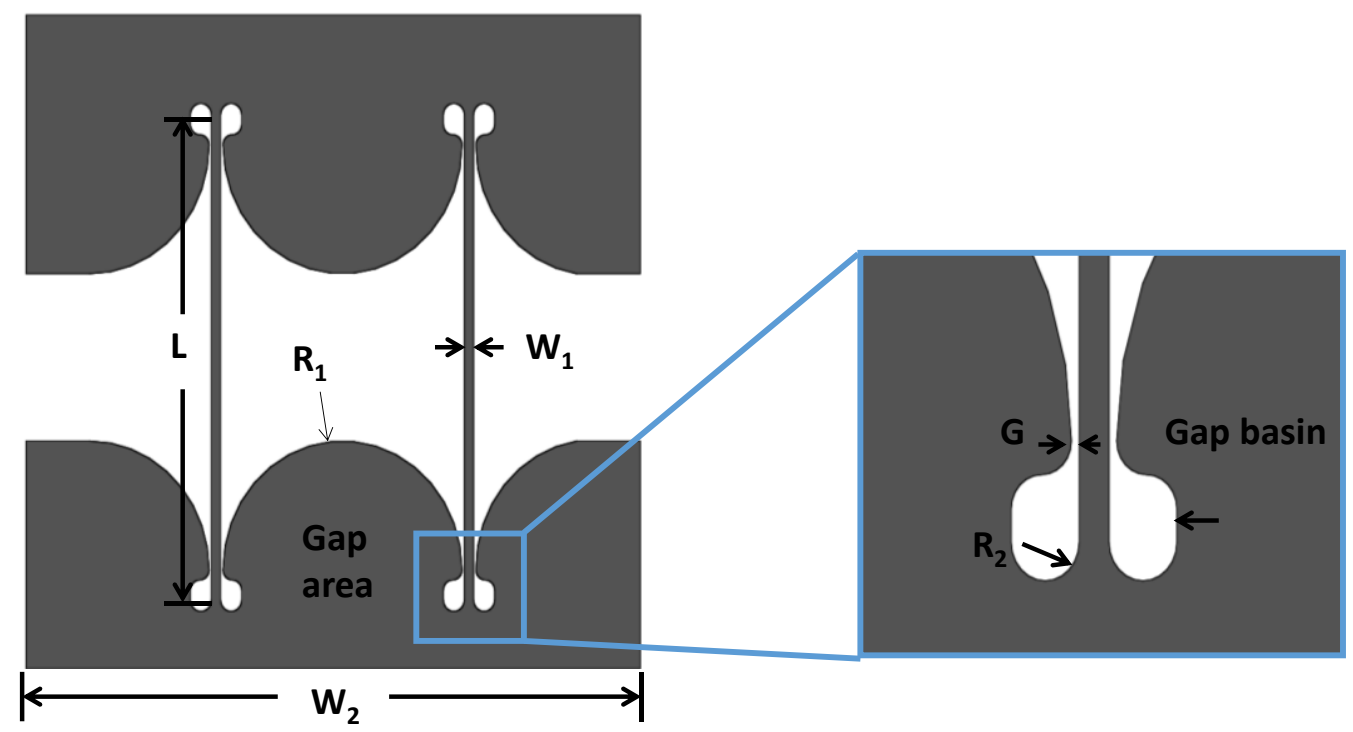

Figure 3 Definition of key geometric parameters in the test structure.

Using a range of radii, one could find the smallest radius part would break or plastically deform and the largest radius part would not. More parts would be manufactured that were larger than the smallest radius until a radius value was found that does not break/deform the beam, but is only slightly larger than a radius which did break the beam. This would provide a lower bound for the ratio $E / S_{y}$. The same process would then be repeated starting from a large radius which did not cause failure in the beam, working down to a small enough radius that did cause failure in the beam, this process would confirm the ratio found in the previous step. Using a confirmed value of $E$, a value of $S_{y}$ could then be determined, thus assessing the strength of the material. In process monitoring, it may be sufficient to print just a single size that confirms a minimum required strength.

The values for each of the cam radii used in this work can be found in Table 1 below. The other designs adjust the radius in steps of $10 \%$ of the nominal design E radius. Design H was twentyfive percent larger than design E, design I was fifty percent larger than design E. 
The length of the beam was determined from equation (22).

$$
L=\pi R+\varepsilon
$$

The beam must wrap around ninety degrees of the bottom cam and ninety degrees of the top cam. The factor of $\varepsilon$ was two millimeters and was used to ensure that the beam was long enough to avoid a stress concentration at the base. The height of the parts was determined by the necessary length of the beam. In addition, it was necessary for there to be a space of material between the gap basin and the bottom/top edge of the part, this space was made to be at least seven millimeters in each of the designs. The gap basin was added because when there was no basin in the gap, the

Table 1 - Dimension values for each design of the part in $\mathrm{mm}$. All parts are $2.5 \mathrm{~mm}$ thick, except design I which is $5 \mathrm{~mm}$.

\begin{tabular}{|c|c|c|c|c|c|c|c|}
\hline Design & $\begin{array}{c}\text { Cam } \\
\text { radius } \\
\left(\mathbf{R}_{1}\right)\end{array}$ & $\begin{array}{c}\text { Beam } \\
\text { length }(L)\end{array}$ & $\begin{array}{c}\text { Beam } \\
\text { width } \\
\left(W_{1}\right)\end{array}$ & $\begin{array}{l}\text { Gap } \\
\text { (G) }\end{array}$ & $\begin{array}{c}\text { Base } \\
\text { fillet } \\
\text { radius } \\
\left(\mathbf{R}_{\mathbf{2}}\right)\end{array}$ & $\begin{array}{c}\text { Part } \\
\text { width } \\
\left(\mathbf{W}_{2}\right)\end{array}$ & $\begin{array}{c}\text { Relative } \\
\text { size }\end{array}$ \\
\hline$A$ & 8.98499 & 30.2272 & 1 & 0.25 & 1.13 & 66 & 0.7 \\
\hline$B$ & 10.2686 & 34.2596 & 1 & 0.25 & 1.13 & 66 & 0.8 \\
\hline$C$ & 11.5521 & 38.2921 & 1 & 0.25 & 1.13 & 66 & 0.9 \\
\hline D & 12.8357 & 42.3246 & 1 & 0.25 & 1.13 & 66 & 1 \\
\hline$E$ & 14.1193 & 46.357 & 1 & 0.25 & 1.13 & 66 & 1.1 \\
\hline $\mathrm{F}$ & 15.4028 & 50.3895 & 1 & 0.25 & 1.13 & 66 & 1.2 \\
\hline $\mathrm{G}$ & 23.1043 & 74.5842 & 1 & 0.25 & 1.13 & 100 & 1.8 \\
\hline $\mathrm{H}$ & 30.8057 & 98.7789 & 1 & 0.25 & 1.13 & 140 & 2.4 \\
\hline 1 & 10.2686 & 34.2596 & 1 & 0.25 & 1.13 & 66 & 0.8 \\
\hline
\end{tabular}

smallest that the gap could be made was limited by the feature size of the machine. By adding the basin, the nozzle of the machine could get a much smaller gap as shown in the figure depicting the gap. With this basin, the gap could be made as small as $0.25 \mathrm{~mm}$, which is the value used in each of the designs. The radius of curvature of the deformed beam is described by equation (19). The walls of the gap basin were made curved so that there would be a smooth transition in the walls which would help to prevent additional stress concentrations. The beam width was one millimeter. Table 1 shows each of the dimensions for each size part. The relative size column refers to the size of the given design relative to the nominal size which is design $\mathrm{D}$.

\section{Test Procedures}

Test components were fabricated from ABS filament on one of four thermal extrusion machines (Stratasys Dimension 768, Stratasys uPrint, Makerbot X2, and a custom-built RepStrap machine utilizing RepRap electronics). Replications of each part were produced with identical printing files to avoid any extra variations in the part. After printing, each part was weighed and the width of the flexible segments was measured using calipers. The weight and measured beam widths were used in the estimates of the strain and elastic modulus of the samples.

On the RepStrap components, the effective fill fraction was adjusted by changing the filament diameter setting in the control software to simulate the effect of variation in the filament diameter 
on the effective mechanical properties of the part. The parts were printed with settings of 2.9, 3.1, and $3.2 \mathrm{~mm}$ while the actual filament diameter varies between 2.9 and $3.0 \mathrm{~mm}$. These variations in fill factor were changed to help us test how feed stock with slightly varying diameter can affect the mechanical properties of the printed parts.

In addition to the test geometries described above, tensile and bending specimens were also prepared using the same printing parameters on both the Dimension and Custom-built RepStrap machines. Tensile and three point bend tests were conducted following ASTM standards D638-03 and D790-03 respectively.

A variety of methods exist for obtaining the vibrational response of a plucked part. For our tests, an inexpensive piezoelectric speaker was placed beneath the component, then clamped to a rigid structure (Figure 4). As the component vibrated the piezoelectric device output a voltage proportional to the force exerted by the oscillating free end. The piezoelectric speaker was then connected to an oscilloscope which provided the voltage vs. time response of the plucked part. If the component is metal and has a higher vibrational frequency, this test can be conducted with items as simple as a hammer and a microphone. A smart phone or tablet with an accelerometer can also be used. By placing the part on the device and striking it, the accelerometer can pick up the vibrations.
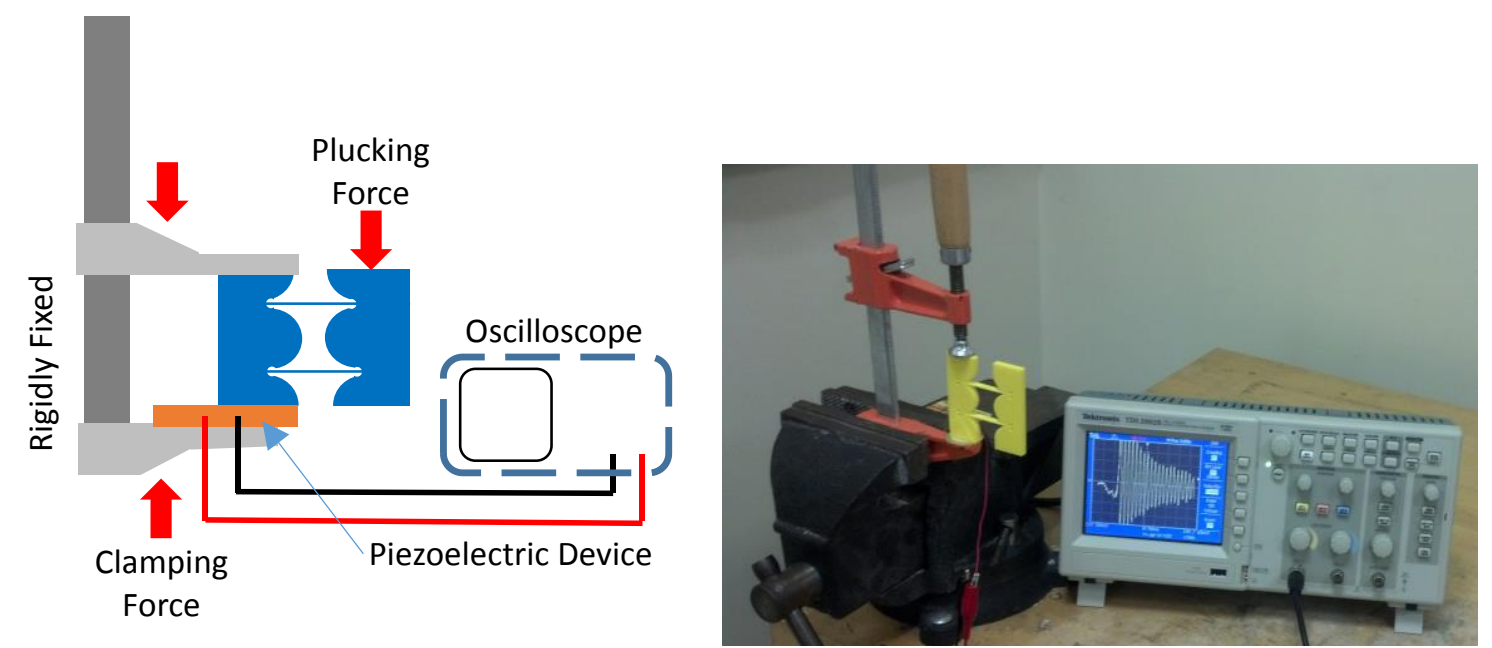

Figure 4 Schematic diagram (left) of test setup (right) for measuring the vibrational frequency of a plucked part.

The plastic strain of the samples was evaluated by deforming the beams around the cam surfaces and then removing the loads. These loads were applied by hand while the part lay horizontal on a flat surface. During deformation, the samples were visually monitored to verify that they remained in contact with the cam surface. The deformed position was maintained for less than five seconds before being released. The parts were gently vibrated to reduce the impact of friction on the final position of the component. The residual horizontal offset between the top and bottom of the structure was then measured to the nearest $0.5 \mathrm{~mm}$ as an indication of the relative plastic deformation of the structures. These tests were done on samples A-H which were produced on the Dimension machine. The residual offset distance and the measured beam widths were used to calculate the residual strain using equation (17). Results are based on one sample of each part size. 
Vibration modulus testing was also conducted on the RepStrap specimens as well as on the Dimension 768 machine. These results were compared against tensile and three point flexural tests conducted on an 858 Material Testing System (MTS). The results for the Dimension 768 were also compared with the manufacturer's specifications. The test specimen for the tensile and flexural tests produced by the RepStrap used layer heights of 0.5 millimeters thick and an infill angle of 45 degrees. The samples produced by the commercial Dimension 768 machine used layer heights of 0.25 millimeters. The infill angle formed by the Dimension 768 machine is unknown, however it can be assumed that it has been optimized to increase the strength of the part. Both the tensile and flexural test specimens were 1 millimeter thick, the same thickness as the beam width for the test structure.

\section{Simulation}

Finite Element Analysis (FEA), modal analysis was performed on the design geometry to compare to the measured vibration frequencies. The simulation assumed ABS properties of $\mathrm{E}=2.0 \mathrm{GPa}$ and a density of $1.02 \mathrm{~g} / \mathrm{cm}^{3}$.

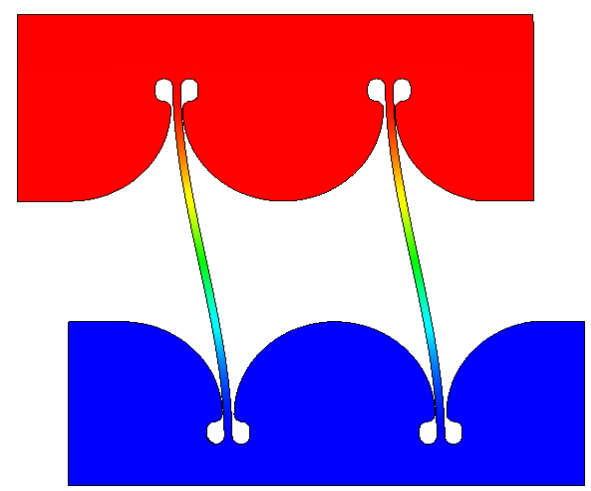

Figure 5 Mode one vibrations as shown in Solidworks FEA

The resulting mode shape is shown in Figure 5Figure 5. As Table 2 shows, the difference between the measured frequencies and the simulated frequencies was relatively small for most of the parts. The exceptions are Parts G, H, and I. Parts G and H were the largest parts and the measured frequency appears to be the third mode. The FEA predicted third mode frequencies on parts $\mathrm{G}$ and $\mathrm{H}$ of $15.225 \mathrm{~Hz}$. and $7.61 \mathrm{~Hz}$. respectively. The difference between measured values and FEA predictions of the third mode is $15.42 \%$ and $4.58 \%$ respectively. The difference between measured values and FEA for part I was attributed to dimensional errors from manufacturing. When the results were corrected for part mass and beam width, the modulus of elasticity estimation was consistent with other parts. Given the close match between the measured and simulated frequencies

Table 2 Frequency values for each part from vibrations tests and FEA in $\mathrm{Hz}$.

\begin{tabular}{|c|c|c|c|c|c|c|c|c|c|}
\hline & Part A & Part B & Part C & Part D & Part E & Part F & Part G & Part H & Part I \\
\hline $\begin{array}{c}\text { Measured } \\
\text { values }\end{array}$ & 41.6 & 35.1 & 30.9 & 24.9 & 22.4 & 16.7 & 18.0 & 8.0 & 18.7 \\
\hline FEA & 39.5 & 32.6 & 28.1 & 23.2 & 21.2 & 18.1 & 7.2 & 3.8 & 33.0 \\
\hline Difference & $5.1 \%$ & $7.1 \%$ & $8.9 \%$ & $6.7 \%$ & $5.4 \%$ & $-8.3 \%$ & $60 \%$ & $52 \%$ & $-76.3 \%$ \\
\hline
\end{tabular}


of the other parts, it is likely that the measured frequency for parts $\mathrm{G}$ and $\mathrm{H}$ were in fact the third vibrational mode.

\section{$\underline{\text { Results }}$}

Table 3 Specimen production scope

\begin{tabular}{|c|c|c|c|c|}
\hline AM Machine & $\begin{array}{c}\text { Specimens } \\
\text { Produced }\end{array}$ & $\begin{array}{c}\text { Alignments } \\
\text { Produced }\end{array}$ & $\begin{array}{c}\text { Number of } \\
\text { Tests }\end{array}$ & $\begin{array}{c}\text { Average Std } \\
\text { Dev }(\mathrm{Hz})\end{array}$ \\
\hline Dimension 768 & $\mathrm{A}-\mathrm{I}$ & $\mathrm{XY}, \mathrm{ZX}$ & 46 & 0.24 \\
\hline uPrint & $\mathrm{I}$ & $\mathrm{XY}, \mathrm{XZ}, \mathrm{YZ}, \mathrm{ZX}$ & 36 & 0.36 \\
\hline RepStrap & $\mathrm{I}$ & $\mathrm{XY}$ & 27 & 0.11 \\
\hline Makerbot X2 & $\mathrm{I}$ & $\mathrm{XY}$ & 9 & 0.20 \\
\hline
\end{tabular}

The measured frequency data generally showed excellent repeatability for each individual part as seen in Table 3. The notable exceptions are parts G-1 and H-1. However, these samples appear to have been vibrating in multiple modes as discussed above.

The relationship of equation (10) was applied to the samples using the nominal (as-designed) density and FEA predictions of vibrational frequency to estimate the effective modulus of elasticity for each combination of geometry and machine tested. The resulting vibration frequency calculations are grouped by machine and printing parameters and summarized in Table 3 compared to the bending and tensile test data. The vibration data results were consistent with the values obtained by traditional tensile and bending methods. The standard deviations obtained from the RepStrap system were comparable in both cases. The high standard deviations in specimens $\mathrm{G}$ and $\mathrm{H}$ are attributed to measuring additional modes of vibration as also seen in Table 2 . However, the parts produced on the commercial Dimension 768 machine produced more repeatable modulus measurements using the flexural and bending tests than was achieved with the vibration method.

In order to determine how the printing orientation effected the modulus, the Stratasys uPrint and Dimension 768 were used to print the test structure in various orientations. The natural frequency was then recorded and used to find the modulus of elasticity as summarized in Figure 7 . There is a close resemblance with the manufacturer's data for the specimens that were printed on-edge or flat on this print bed. The modulus values are expected to be similar as the deposition lines are oriented parallel with the print bed, unlike the upright orientation. However, the test specimens that were printed in the non-ideal orientation, standing up on the build platform in the Z-direction produced a significantly lower modulus as has been reported previously by Stratasys [15] and others [16]. 
By changing the effective filament diameter for the RepStrap in the control software to simulate a feed stock that was produced out of specification we are able to study effects on the mechanical properties. As the effective diameter of the filament was decreased, the AM machine increased the amount of material used, which produced a heavier part with a higher frequency and modulus. Since the natural frequency is related to the square root of the stiffness divided by the mass (equation 4), this is an indicator that the stiffness of the part has increased as well. A small standard deviation indicates good repeatability in manufacturing and measurement.

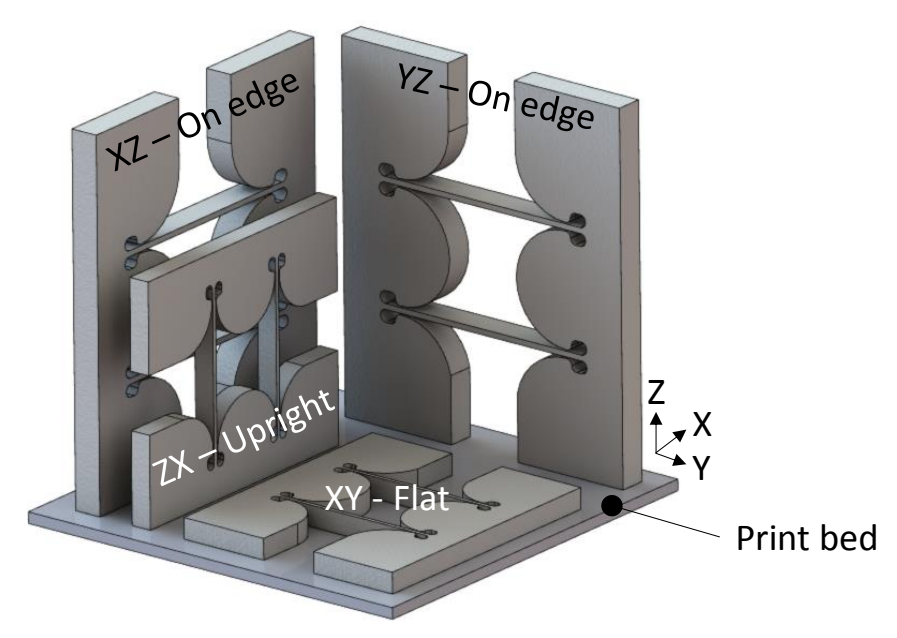

Figure 6 Printing orientations, parts not to scale.

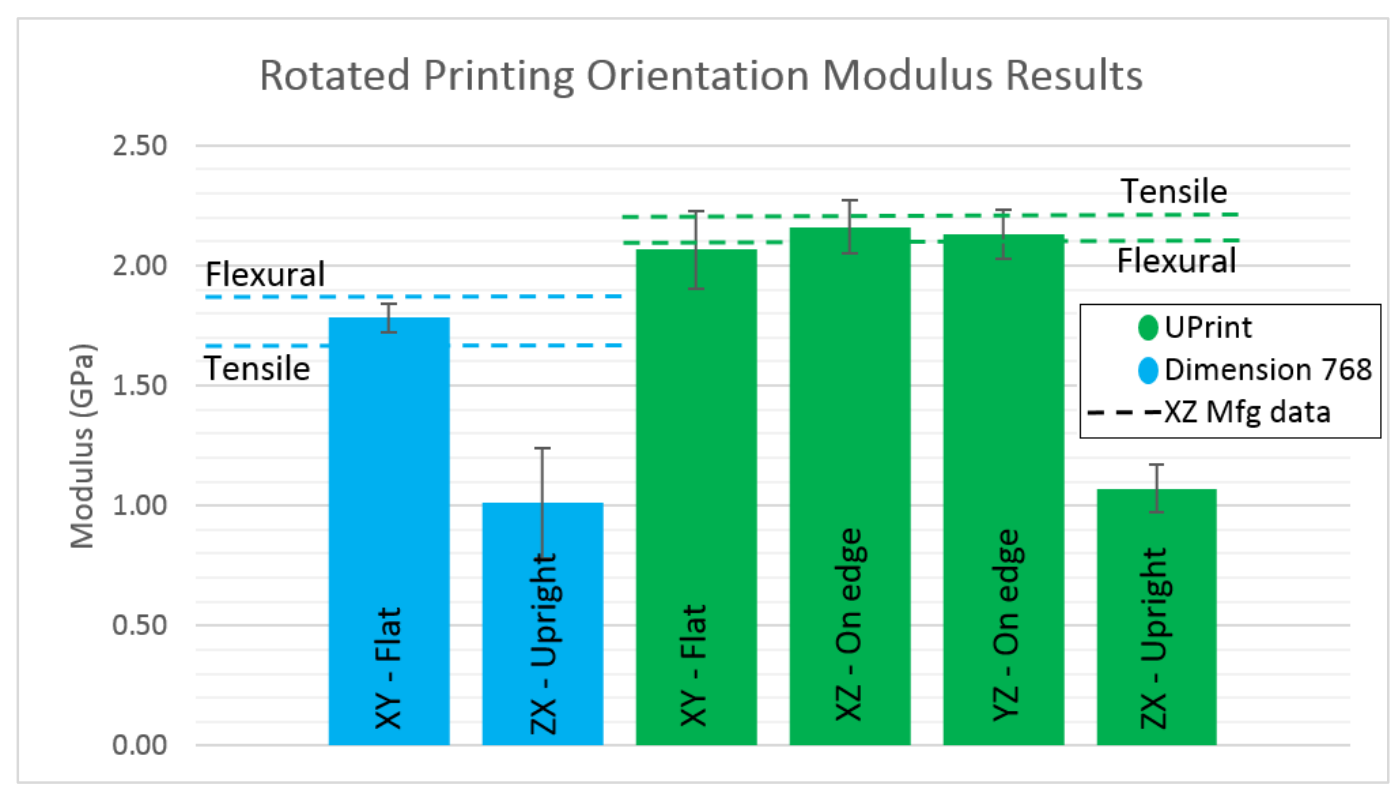

Figure 7 Modulus values measured by vibration method of test structures printed in various orientations.

The measured vibrational modulus from the RepStrap was well below both the flexural and tensile modulus found from material testing system. However, they were closer to the results of the tensile tests than the flexural tests. This could be due to variations in the beam thickness throughout each part as well as other non-uniformities inherent in lower end 3D printers. 


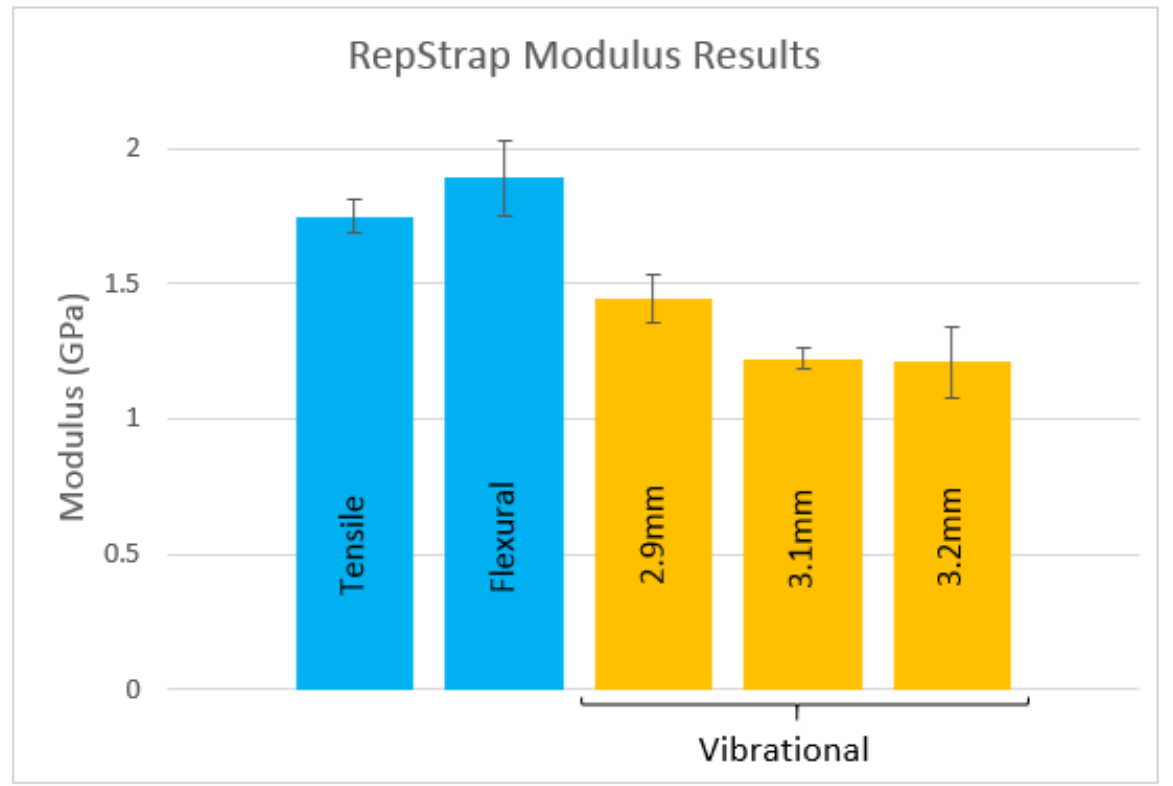

Figure 9 Mechanical properties from various tests. The vibrational tests were conducted with varied effective filament diameter.

The parts used in the strength test exhibited yielding but not fracture. Most of the tested parts had measurable deformations, i.e. the top portion of the part had translated a measurable amount from its original symmetric configuration as shown in Figure 1 (left).

shows the deformations and calculated applied and residual strains for the tested parts. The results are consistent with the elastic behavior for specimens $\mathrm{G}$ and $\mathrm{H}$, and a combination of elastic and plastic behavior for specimens A-F. The results are consistent with an elastic strain limit of approximately $2.1 \%$ as seen in Figure 8 . This compares favorably to the elastic limit of $2.2 \%$

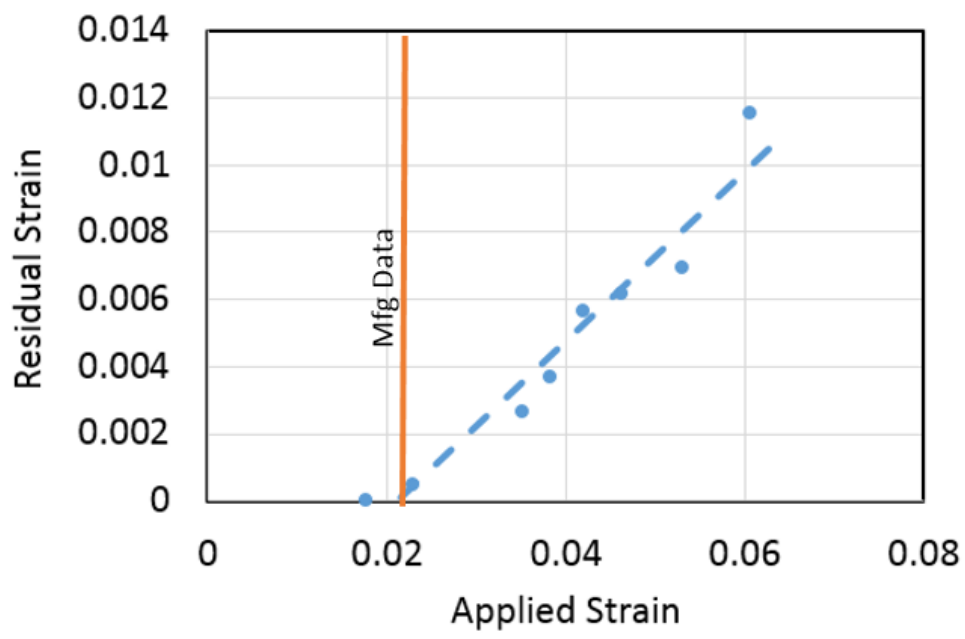

Figure 8 Residual plastic strain as a function of applied elastic strain. The $x$-intercept represents the onset of plastic strain. 
$\left(\varepsilon_{\text {elastic limit }}=S_{y} / E\right)$ that would be estimated from the flexural data reported by Stratasys $\left(S_{\text {flex }}=\right.$ $\left.41 \mathrm{MPa}, \mathrm{E}_{\text {flex }}=1,834 \mathrm{MPa}\right)$ [17].

\section{Discussion}

The modulus of bulk raw material is generally known, but the effective modulus of the printed structure can deviate significantly due to voids, under-filling, over-filling in the structure, anisotropy in the deposited material and other miscellaneous variations [4]. The yield strength $\left(S_{y}\right)$ can also vary with the printing process due to the effects of temperature on the material and the bonding between features. There is also uncertainty in the actual value of $c$ due to the tolerances of the manufacturing process. These differences will affect both the vibration frequency and the plastic deformation. Since these effects are not easily isolated, it may be difficult to isolate the cause of a change. However, the measurements can be used to monitor for process changes that affect the density, finished dimensions, feature bonding, and material properties.

As each component is created by AM processes, the orientation or position of the part can vary the amount of void space or dimensional inaccuracies within a part. The vibrational analysis yields natural frequencies that vary little between tests of the same part but have a larger variation between individual parts with similar geometries. This would imply that the measuring system is repeatable, but each identical component produced from AM has varying degrees of inaccuracies. By simply measuring the weight of a component, a rough estimate of the degree of inaccuracies can be found. However this does not take into account how this would affect the mechanical properties of the component, namely elastic modulus. By using the equations found in the Theory section we can then extract the estimated modulus values.

During the initial deformation of the test samples, audible pops were often observed for the small cam radii. Inspection of the specimens frequently showed delamination of the beams in these cases. Thus, these thin beams appear to be failing in shear rather than tension. This would be surprising for an isotropic material as the shear stress should be small under the expected load condition. This may indicate that the loading does not fully reach the idealized full bending condition. However, there is a concentration of shear as the beam transitions between the curvatures of the two cams. Additionally, the bonding between adjacent deposition lines is known to be weaker than the native polymer. This is especially possible in thin beams made without fill in which the software that defines the AM machine's route may be prioritizing accuracy of external dimensions over mechanical strength.

While the vibrations were measured using an oscilloscope in this work, the speaker output could be read by more commonly available computer inputs such as a microphone jack on a cellular phone or laptop. We have also demonstrated that we can detect the vibrations using the accelerometers built into many tablets and smart phones. Thus, these methods could be adapted to the use by DIY testers with minimal expense. They could also be integrated as test coupons in production manufacturing without the need to use expensive testing machines for routine process monitoring. However, to be useful in process monitoring, the test method needs to be highly reliable. While these results were promising, more evaluation is necessary to assess whether the method is sufficiently accurate to use in these applications. While the variation in the measurements was significant in the initial tests, it is unclear whether this is due to variation in the test methods or the parts being tested. 


\section{Conclusion}

A simple test geometry has been presented that facilitates assessments of strain limits of the material and the elastic modulus. This method utilizes flexible elements and a circular cam to apply a uniform strain across the flexible elements utilizing simple hand loading. A series of different sized elements can be used to identify the elastic strain limit of the printed structures. Additionally, the natural frequency of the structure provides an estimate of the structure's elastic modulus. The required equipment is readily available to DIY users. The method could also be useful for monitoring the stability of production processes.

A series of components have been manufactured using thermal extrusion and the test methods were applied to them. When applied to a series of parts with different bend radii, the measured results provided an estimate of the elastic strain limit of the material. The elastic modulus estimates showed consistent predictions for components produced on the same machine even when the key geometric parameter (cam radius) varied. The method also showed an ability to detect the effects of a printing parameter on the effective modulus of elasticity of the printed structure. These results show that the outcomes, particularly the frequency, are sensitive to variations between parts. Further work is needed to identify the sensitivity of these measurements to other sources of process variation.

\section{$\underline{\text { References }}$}

[1] I. Gibson, D. W. Rosen and B. Stucker, Additive Manufacturing Technologies: Rapid Prototyping to Direct Digital Manufacturing. New York: Springer, 2010.

[2] F. Abe, E. Costa Santos, Y. Kitamura, K. Osakada and M. Shiomi, "Influence of forming conditions on the titanium model in rapid prototyping with the selective laser melting process," Proc. Inst. Mech. Eng. Part C, vol. 217, pp. 119-126, 2003.

[3] S. Ahn, M. Montero, D. Odell, S. Roundy and P. K. Wright, "Anisotropic material properties of fused deposition modeling ABS," Rapid Prototyping Journal, vol. 8, pp. 248-257, 2002.

[4] M. S. Hossain, J. Ramos, D. Espalin, M. Perez and R. Wicker, "Improving tensile mechanical properties of FDM-manufactured specimens via modifying build parameters," in Solid Fabrication Symposium, Austin, TX, 2013, .

[5] P. Kulkarni and D. Dutta, "Deposition strategies and resulting part stiffnesses in fused deposition modeling," J. Manuf. Sci. Eng. Trans. ASME, vol. 121, pp. 93-103, 1999.

[6] K. Thrimurthulu, P. M. Pandey and N. V. Reddy, "Optimum part deposition orientation in fused deposition modeling," International Journal of Machine Tools \& Manufacture, vol. 44, pp. 585-94, 05, 2004.

[7] K. P. Cooper and R. F. Wachter, "On making additive manufacturing a production technology," in 2011, pp. 1593.

[8] J. Xiong and G. Zhang, "Online Measurement of bead Geometry in Gmaw-based Additive Manufacturing Using Passive Vision," vol. 24, pp. 115103 (7 pp.), 11, 2013. 
[9] K. Zeng, N. Patil, H. Gu, H. Gong, D. Pal, T. Starr and B. Stucker, "Layer by layer validation of geometrical accuracy in additive manufacturing processes," in 24th International Solid Freeform Fabrication Symposium - an Additive Manufacturing Conference, SFF 2013, August 12, 2013 - August 14, 2013, pp. 76-87.

[10] N. Akhter, H. Jung, H. Chang and K. Kim, "Determination of elastic modulus of a beam by using electronic speckle pattern interferometry," Opt. Laser Technol., vol. 41, pp. 526-529, 2009.

[11] R. M. Digilov, "Flexural vibration test of a cantilever beam with a force sensor: fast determination of Young's modulus," European Journal of Physics, vol. 29, pp. 589-97, $05,2008$.

[12] R. M. Digilov and H. Abramovich, "Flexural Vibration Test of a Beam Elastically Restrained at One End: A New Approach for Young's Modulus Determination," Advances in Materials Science \& Engineering, pp. 329530 (6 pp.), 2013.

[13] ASTM Standard E1876-01, "Standard test method for dynamic young's modulus, shear modulus, and poisson's ratio by impulse excitation of vibration," ASTM International, West Conshohocken, PA, 2006.

[14] R. J. Roark and W. C. Young, Roark's Formulas for Stress and Strain. New York: McGrawHill, 1989. (2014). APSplus-P430. Available: http://www.stratasys.com/ /media/Main/Secure/Material\%20Specs\%20MS/Fortus-MaterialSpecs/Fortus-MS-ABSplus-01-13-web.pdf.

[16] S. V. Raut, V. S. Jatti and T. P. Singh, "Influence of built orientation on mechanical properties in fused deposition modeling," in 2014 International Mechanical Engineering Congress, IMEC 2014, June 13, 2014 - June 15, 2014, pp. 400-404.

[17] (). Dimension P400 Material Properties [Dimension P400 Material Properties]. Available: https://store.amtekcompany.com/products.php?product=Dimension-Standard-ABS-ModelMaterial-\%25252d-P400. 